Voix et Images

voixetimages

\title{
Glissements déictiques et récit schizophrénique dans Après la boue de Gilbert La Rocque
}

\section{Julie LeBlanc}

Volume 15, numéro 3 (45), printemps 1990

Gilbert La Rocque

URI : https://id.erudit.org/iderudit/200853ar

DOI : https://doi.org/10.7202/200853ar

Aller au sommaire du numéro

Éditeur(s)

Université du Québec à Montréal

ISSN

0318-9201 (imprimé)

1705-933X (numérique)

Découvrir la revue

Citer cet article

LeBlanc, J. (1990). Glissements déictiques et récit schizophrénique dans Après la boue de Gilbert La Rocque. Voix et Images, 15(3), 352-362.

https://doi.org/10.7202/200853ar d'utilisation que vous pouvez consulter en ligne.

https://apropos.erudit.org/fr/usagers/politique-dutilisation/ 


\title{
Glissements déictiques et récit schizophrénique dans Après la boue de Gilbert La Rocque
}

\author{
par Julie LeBlanc, université Carleton
}

\begin{abstract}
L'autre, c'est le lieu étranger d'où émane tout discours [...] le lieu où est renvoyé toute subjectivité. 1
\end{abstract}

Que dire d'un roman dont la trame narrative ne cesse d'être perturbée par de nombreux changements de registre et dont la diégèse est composée d'une suite de segments rétrospectifs dénués de tout ordre? Comment cerner ces deux phénomènes qui résument ce qu'il y a d'essentiel dans Après la boue ${ }^{2}$ de Gilbert La Rocque ? C'est en recourant à la deixis que nous nous proposons de décrire les mécanismes qui sous-tendent la structure narrative de ce roman. En privilégiant le jeu des glissements déictiques ${ }^{3}$ inscrit tout au long de cette œuvre romanesque, notre but sera non seulement de préciser dans quelle mesure ce phénomène sert à marquer chez l'héroïne l'acheminement vers la folie, mais également de tenter d'élaborer un modèle d'énonciation du récit schizophrène fondé sur les principaux éléments de la deixis.

La valeur de la notion de déictique, pour l'analyse de textes littéraires, provient surtout du fait qu'elle peut prendre en charge de nombreux éléments d'ordre narratif. Tout en étant aptes à rendre compte des rapports entre les "protagonistes" du discours (le locuteur et son interlocuteur) et à préciser le cadre spatio-temporel de l'activité d'énonciation (ici/ailleurs, maintenant/non-maintenant), les indicateurs de la deixis sont également susceptibles de prendre en

1 Catherine Backès-Clément, "(Lacan) Jacques", la Grande Encyclopédie Larousse, tome II, Paris, Larousse, 1974, p. 6897.

2 Gilbert La Rocque, Après la boue, Montréal, Québec/Amérique, 1981. Ce roman fut d'abord publié aux Éditions du Jour en 1972.

3 Cette expression est emprunté à Pierre Van den Heuvel, Parole Mot Silence, Paris, José Corti, 1985, p. 98. Le concept de "glissements déictiques " décrit très bien les changements de formes pronominales ou, si nous préférons, les glissements du pronom de la première personne à celui de la troisième personne inscrits tout au long du récit de Gabrielle, l'héroïne d'Après la boue. 
charge tout un ensemble de données (instance narrative, rapport du narrateur à son énoncé, distance énonciative) et d'introduire au sein du langage une subjectivité indéracinable 4 .

L'ampleur du champ couvert par la deixis (formes pronominales, références spatio-temporelles, démonstratifs), de même que les problèmes narratifs propres à Après la boue exigent un resserrement de notre problématique qui nous incite à privilégier les déictiques pronominaux. Sans trop multiplier les citations bien connues, nous adopterons donc dans cette étude l'optique d'Émile Benveniste et de Catherine Kerbrat-Orecchioni selon qui les pronoms personnels sont non seulement le premier point d'appui pour cette mise au jour de la subjectivité dans le langage ${ }^{5}$, mais également un des lieux d'ancrage les plus manifestes de la subjectivité langagière ${ }^{6}$. C'est dans la mesure où la deixis pronominale peut rendre compte de la présence du sujet dans son énoncé, de la relation du locuteur à l'interlocuteur et de la disposition du sujet vis-à-vis du message énoncé, qu'elle doit être perçue comme une des données linguistiques les plus susceptibles de décrire la spécifité de l'instance schizophrénique inscrite au sein de ce roman de Larocque 7 .

\section{Glissements déictiques et récit schizophrénique}

Comme nous l'avons déjà signalé, ce qui nous invite à faire appel au phénomène de la deixis, c'est la complexité narrative d'Après la boue. Le roman met en scène trois récits distincts, trois instances narratives et trois sujets de focalisation. Il y a, d'une part, le récit à la première personne dans lequel Gabrielle, l'héroïne, tente de présenter par d'incessants retours en arrière ses expériences traumatisantes: J'ai trente ans, c'est jeune, c'est la vie qui commence pour moi. [...] Mais on vit on a vécu, et il n'en reste pour ainsi dire plus rien. Rien que soi au bout de son ridicule fil de souvenirs... (p. 16) D'autre

4 John Lyons, Sémantique linguistique, Paris, Larousse, 1980, p. 269.

5 Émile Benveniste, Problèmes de linguistique générale, Paris, Gallimard, 1966, p. 262.

6 Catherine Kerbrat-Orecchioni, l’Énonciation. De la subjectivité dans le langage, Paris, Armand Colin, 1980, p. 33.

7 C'est le processus de dédoublement auquel se prête la narratrice, dans son évocation de certaines expériences traumatisantes, qui nous incite à faire appel au concept de schizophrénie dans notre analyse d'Après la boue. En parlant de soi à la troisième personne, l'héroïne manifeste à l'égard des événements évoqués dans son récit un certain "détachement " qui met en cause son rapport à la réalité. Dans "Une rhétorique efficace", Gilles Dorion recourt également au concept de schizophrénie pour décrire la structure narrative d'Après la boue. Comme l'explique Dorion, ces nombreux fragments du roman dans lesquels l'héroine, Gabrielle, semble parler d'elle-même à la troisième personne peuvent être perçus comme des marques évidentes de schizophrénie, bien plus que de changement véritable de narratrice ou de focalisation narrative. Gilbert La Rocque. L'écriture du rêve, Montréal, Québec/Amérique, 1985, p. 80. 
part, il y a le récit dans lequel le narrateur, appelé omniscient selon la terminologie courante anglo-saxonne, déploie une maîtrise totale à l'égard de l'histoire, des pensées et des sentiments de l'héroïne: Elle prit sa valise et sortit dans le corridor, pensant à toute vitesse mais bien sûr dérapant et glissant d'une pensée à la suivante sans pouvoir rien retenir ou utiliser [...]. (p. 183) Enfin, il y a le récit schizophrénique de Gabrielle qui raconte à la troisième personne certains événements de sa vie, comme s'il s'agissait de l'histoire d'une autre: je m'éveillai comme tous les matins [...] je m'éveillai très lentement, je flottais Gaby flottait dans un demi-sommeil [...]. (p. 24)

Les oscillations de la trame narrative, l'ambigüté des instances locutrices, l'imprécision du contexte spatio-temporel proviennent tous, dans ce roman, des indicateurs pronominaux. C'est la catégorie de la personne qui veille à l'organisation du récit, ce sont les pronoms qui déterminent le rôle des participants de l'acte d'énonciation, ce sont des indicateurs pronominaux dont dépendent les autres formes de la deixis. Bref, avec Après la boue, nous sommes en présence d'une œuvre où le récit est mis en cause par de nombreux changements de registres narratifs, où la spécificité des instances narratives se trouve compromise par l'absence de signes typographiques et lexicaux et où le sujet est subverti par la présence de nombreuses voix narratives qui cherchent toutes à s'approprier l'appareil formel du discours ${ }^{8}$. En privilégiant ainsi tout ce qui se passe sur le plan de l'instance narrative, nous arriverons non seulement à mieux comprendre le statut des différents sujets d'énonciation et à saisir la nature obsessionnelle du récit schizophrénique, mais nous parviendrons également à mettre à nu les forces dynamiques d'une œuvre vouée à décrire l'aliénation psychique d'un être aux prises avec l'impossible choix entre son monde et son moi ${ }^{9}$.

Cette phrase résume ce qu'il y a d'essentiel dans Après la boue. Par une série de fragments rétrospectifs, Gabrielle tente de raconter son histoire, de reconstituer le fil des souvenirs qui lui déferlent dans la tête (p. 16). Cette remontée dans les eaux noires de son passé (p. 75) est actualisée par un jeu narratif oscillant entre les pôles de la personne subjective (le «je») et de la non-personne (le "elle»)10. Les changements de registre narratif produits par l'alternance des formes grammaticales donnent liéu à un récit schizophrénique dans lequel l'héroïne évoque l'histoire de sa vie comme s'il s'agissait de celle d'une autre. La reconstitution du sujet s'effectue donc dans ce roman par une figure de dédoublement où la "personne" et la "nonpersonne", où le moi et l'autre peuvent être perçus comme désignant les deux faces opposées d'une image de soi: partie saine et partie

8 Émile Benveniste, op. cit., p. 239.

9 Paul-Claude Racamier, les Schizophrènes, Paris, Payot, 1980, p. 97.

10 Émile Benveniste, op. cit., p. 232. 
folle ${ }^{11}$. C'est que ce changement d'une instance narrative à l'autre semble traduire chez Gabrielle l'existence de deux attitudes essentiellement opposées vis-à-vis de son passé: d'une part un désir de capter ce vécu, de l'assumer, et d'autre part, un effort pour se détacher de la réalité mise en cause, pour s'en dissocier. Ce jeu des glissements déictiques, qui servent en quelque sorte à marquer chez l'héroïne l'acheminement vers la folie, est introduit dès les premières pages du roman:

[...] on n'a pas le droit de dire non ou d'être méchante il faut toujours obéir! et elle ne peut pas bouger c'est défendu, mais ça n'a pas d'importance, il fait chaud et il fait très chaud, voyez comme mes cheveux brûlent je suis torche cierge magnifique [...] je n'ai pas peur, nọn, je n'ai rien à craindre, Gabrielle chante en descendant l'escalier étroit qui mène à la cave [...] elle est toute nue je suis toute nue mais je ne suis pas fatiguée [...]. (p. 15)

\section{L'ambiguïté des instances narratives}

Cet extrait présente explicitement les deux phénomènes dont découle la complexité narrative d'Après la boue. L'absence d'indicateurs d'aiguillage 12 d'ordre typographique (italiques, guillemets, alinéas) et lexical (dit-elle, pensa-t-elle) contribue à gommer le fonctionnement des instances narratives. Parce que le changement de l'instance "omnisciente" à celle du "je» n'est pas clairement marqué, la singularité des différentes voix narratives se trouve compromise. L'exclusion des indicateurs typographiques et lexicaux tout au long du roman a non seulement pour effet d'assimiler deux plans d'énonciation qui devraient normalement être nettement distingués, mais également de pervertir la spécificité des diverses instances narratives.

Les nombreuses ambiguïtés inscrites tout au long d'Après la boue proviennent surtout du manque de précision par rapport à la relation entre le narrateur et le personnage. D'un segment du roman à l'autre, il est en principe difficile de préciser si les allusions à la première et à la troisième personne appartiennent à l'instance "autodiégétique» (ou récit schizophrénique), ou s'il ne s'agit pas plutôt de fragments de discours rapportés, évoqués par l'instance omnisciente: je suis une ruine oh que je me déteste! et elle regarde encore les comprimés, et elle comprend de moins en moins, non je ne le ferai pas [...] et tout cela pulsait sauvagement dans sa tête, dans ma tête! (p. 182) Les glissements d'une instance à l'autre sont nettement marqués dans cet extrait par l'emplacement des formes pronominales et des adjectifs possessifs. Le va-et-vient entre les indicateurs de la première et de la

11 Paul-Claude Racamier, op. cit., p. 75.

12 Expression empruntée à François Jost, "Le Je à la recherche de son identité", Poétique, no 24, 1975, p. 481. 
troisième personne donne lieu, dans la trame narrative, à des changements de registre qui sont clairement perceptibles et qui ne peuvent être négligés. Toutefois, l'ambiguïté qui provient de ce flottement des instances narratives se trouve accentuée par le fait que l'on parvient difficilement à déterminer qui est le discours de qui tout en sachant que plusieurs discours s'entremêlent ${ }^{13}$.

Outre cette absence d'indicateurs d'aiguillage, ce qui contribue également à rendre ambiguë la structure narrative d'Après la boue, c'est du jeu des glissements de la première à la troisième personne que nous provient le récit schizophrénique de Gabrielle. Ce jeu de dédoublement dans lequel l'héroïne feint de parler d'une autre tout en parlant de soi donne lieu à de nombreux flottements et à de nombreuses perturbations. C'est par ce déplacement d'une instance narrative à l'autre que s'opère, chez l'héroïne, le désinvestissement du «je» et que se manifeste le conflit entre le «moi» et l'autre, entre la préservation narcissique et l'aspiration antinarcissique ${ }^{14}$.

Et $\mathrm{j}$ 'étais allée Gaby était allée ce matin-là travailler même si elle avait la tête comme un atelier de forge, marteaux cognant aux tempes... [...]. La veille javais encore pris mes comprimés de somnifere, [...] je m'éveillais très lentement, je flottais Gaby flottait dans un demi-sommeil, un état de mort latente, pas encore éveillée mais ne dormant quand même plus, comme léthargique tout simplement... puis elle sentit qu'elle était maintenant éveillée [...]. (p. 23-24)

Tout en instituant des changements de registre dans la trame narrative, tout en ébranlant le statut du sujet parlant, l'essentiel de ces glissements de personnes (jétais allée / Gaby était allée même si elle avait la tête comme un marteau, je flottais / Gaby flottait [...] puis elle sentit) provient du fait qu'ils sont à la base de l'instance schizophrénique. Dans ce jeu de dédoublement, par l'entremise duquel Gabrielle tente de revivre les séquences importantes de sa vie comme s'il s'agissait de la vie d'une autre, c'est la relation du «moi " à la réalité qui est mise en cause. Quelle que soit l'expression employée pour décrire le processus de dépersonnalisation auquel se prête la narratrice, clivage du moi (Pontalis/Laplanche), désinvestissement $d u$ je (Racamier), il importe de reconnaître que ce flottement des instances narratives (“je” à elle, "je» à Gabrielle) est le lieu où se rencontrent et se différencient l'image de l'autre et l'image de soi ${ }^{15}$.

En fait, ce glissement d'une personne à l'autre traduit chez l'héroïne deux attitudes conflictuelles vis-à-vis de ses expériences

13 Ibid., p. 482.

14 Paul-Claude Racamier, op. cit., p. 13.

15 Ibid., p. 13. 
vécues: une prise en charge de la réalité en cause et une dénégation de cette même réalité. Cette tentative de rassembler les bribes éparses de son histoire signale de la part de Gabrielle un désir d'inté-. rioriser et pour ainsi dire de faire face à l'horreur et au désespoir de certaines épreuves déchirantes. Toutefois, dans la mesure où l'évocation de ses expériences traumatisantes (les avances sexuelles de son oncle, la violence de son mari, l'avortement de l'enfant qu'il lui a fait, sa culpabilité par rapport à l'aveuglement de son père) n'a lieu qu'à partir d'un état de dédoublement, ce n'est pas une prise en charge de la réalité que l'héroïne effectue, mais plutôt un détachement à l'endroit de la réalité 16 .

- il fait noir dans l'escalier, c'est bien long il faut descendre très longtemps, il y a des chiens enragés qui veulent la mordre, ils ont arraché la robe blanche, elle est toute nue je suis toute nue mais je ne suis pas fatiguée, [...] vous voyez bien que je suis dans mon lit comme tous les bébés morts, et Gabrielle est étendue nue sur le lit et le lit est très grand, il fait rouge mais il fait noir et la grande femme est blanche, il fait froid maintenant et la femme a des mains de terre gelée [...]. (p. 15)

Outre les nombreux glissements déictiques, on relève dans elle est toute nue je suis toute nue [...] vous voyez que je suis dans mon lit [...] et Gabrielle est étendue nue sur le lit, une corrélation de personnalité et de subjectivité ${ }^{17}$ dans laquelle s'opposent de nombreuses instances narratives: "je» à elle, «je» à Gabrielle, "je» à vous. Si nous acceptons que je n'emploie je qu'en m'adressant à quelqu'un, ce sera dans mon allocution un $\mathbf{t u}^{18}$, c'est que nous croyons que par sa nature testimoniale, le récit schizophrénique de Gabrielle présuppose un destinataire. La présence de cette instance est d'autant plus marquée au début de certains fragments rétrospectifs, dans lesquels l'héroïne manifeste un certain désir d'ordonner les maillons de cette longue chaîne de souvenirs décousus. On notera également que la présence du destinataire ne semble se manifester que par rapport à l'évocation de certaines expériences, notamment lés plus angoissantes: Non. Il faut avancer encore... (Je voudrais tout dire) [...] Essayer encore... Autrefois... Non... Tiens... C'est Noël, c'est le réveillon chez grand-mère [...] et Gabrielle est assise sur son oncle Émilien [...]. (p. 16)

Dans le récit, le statut des "protagonistes de l'énonciation" (du locuteur et de l'interlocuteur) est non seulement subverti par les nombreux glissements de formes pronominales, mais l'absence de détails sur le destinataire du récit schizophrénique donne lieu à un

16 J. Pontalis et J.-B. Laplanche, Vocabulaire de psychanalyse, Paris, PUF, 1984, p. 433.

17 Émile Benveniste, op. cit., p. 235.

18 Ibid., p. 260. 
brouillage actanciel qui n'est jamais entièrement résolu. Tout en étant essentiel au récit schizophrénique, l'interlocuteur n'est ni représenté par un personnage ni mentionné explicitement. Son identité est une énigme qui est davantage obscurcie à la fin du roman par la suggestion de la présence de nombreux interlocuteurs:
[...] je ressentais le besoin de raconter mon histoire il fallait que je m'en délivre il fallait que j'en accouche et parler au docteur Narcix ne me suffisait plus et j'accrochais d'autres malades et je racontais l'histoire de Gabrielle, je réinventais l'histoire de Gabrielle et chacune m'écoutait patiemment [...] et je me racontais interminablement j'en avais la bouche sèche [...]. (p. 194)

\section{Le nom propre et le problème de distance narrative}

Comme l'indiquent certains des extraits précités, dans sa tentative de s'expulser de l'histoire qu'elle raconte, l'héroïne recourt également à son nom propre. De façon analogue au pronom de la troisième personne, le nom de Gabrielle permet à l'héroïne de parler de soi en s'absentant de la surface textuelle de son récit. En remplaçant ainsi l'indicateur de la troisième personne, qui est incapable d'individualiser et qui ne désigne en soi spécifiquement rien ni personne ${ }^{19}$, par un nom propre dont la principale fonction est de poser l'individualité et la singularité de l'objet auquel il s'applique ${ }^{20}$, l'héroïne ne fait qu'accentuer son aliénation psychique et intensifier ce désir de se distancier de certaines expériences vécues:

\section{C'est Nöl, c'est le réveillon chez Grand-mère [...] et Gabrielle} est assise sur son oncle Émilien, ils sont tout seuls dans la chambre de grand-mère [...]. (p. 16)

Gabrielle a neuf ans [...] et il a des mains froides et sèches, insectes plats qui voyagent sur et sous la robe de Gabrielle [...]. (p. 17)

[...] les mains de son oncle lui font mal ou lui font peur, c'est la même chose au fond [...] et Gabrielle le laisse écarter ses cuisses fines et elle ferme les yeux de toutes ses forces [...]. (p. 18)

Tout en permettant de désigner, le nom de Gabrielle sert également à individualiser et donc à intensifier cet écart entre le sujet d'énonciation et le sujet de l'énoncé. Cet effet de distanciation, qui est actualisé par les glissements de la première à la troisième personne, est au confluent de toutes les ambiguités ${ }^{21}$ narratives du récit schizophrénique. Ce phénomène d'écart, qui sous-tend la structure narrative du récit de

19 Ibid., p. 260.

20 Jean-Claude Pariente, le Langage de l'individuel, Paris, Armard Colin, 1973, p. 69.

21 Catherine Kerbrat-Orecchioni, op. cit., p. 153. 
Gabrielle, met en place une dialectique du "je " et de l'autre, de la réalité et de lillusion, susceptible de prendre en charge le fonctionnement de l'œuvre entière. Les opérateurs d'individualisation 22 (indicateurs pronominaux, noms propres), qui peuvent servir à indiquer que ce qui est énoncé est plus ou moins pris en charge par le sujet parlant ou à traduire de la part du locuteur un certain degré d'adhésion au message communiqué, sont également disposés à introduire, par un jeu de subversion, un écart entre le sujet et son énoncé. Par l'entremise du pronom de la troisième personne et de son nom propre, l'héroïne parvient à se maintenir à une "distance maximale " de certaines expériences traumatisantes. Tout en traduisant de la part de Gabrielle une certaine disposition à l'égard de son moi de jadis, cette distance exprime également un certain refus de faire face aux motifs de son aliénation psychique:

[...] non... c'est plus tard, beaucoup plus tard, il manque des chaînons, mais il y a ceci, c'était l'an passé, j'étais couchée Gaby était couchée avec son mari Roch cher mari gros chéri soufflant sexe et il lui passe la main sur le ventre comme ça, elle est nue elle voudrait mettre son pyjama et dormir au lieu (mon Dieu!) et justement c'est ce fameux vendredi d'août où il fait si chaud, elle avait tellement mal à la tête! [...] (p. 19)

[...] alors j'étais assise Gaby était assise dans la baignoire vide, elle était toute crispée et avec ses cuisses blanches écartées elle devait ressembler à une grenouille morte [...] Gaby avait dans sa main droite la grande aiguille à tricoter l'aiguille en plastique rouge flexible à souhait, et brutalement c'est la douleur inimaginable douleur explosant comme une mine de fond dans l'océan d'elle et voici du sang ah Seigneur! [...]. (p. 21)

L'essence de ces retours en arrière, c'est qu'ils renvoient tous à des faits maintenus, repoussés et refoulés hors de la pensée de l'héroïne. Dans toutes les évocations traitant des expériences traumatisantes de Gabrielle, le sujet qui parle reste distinct, lorsqu'il parle de lui-même; du sujet de son énoncé ${ }^{23}$. Le récit de la troisième personne, par l'entremise duquel le sujet parle divisé, est dans une certaine mesure le lieu de retour du refoulé. L'évocation de ces souvenirs, qui devrait mener à leur actualisation, est détournée par l'emploi de la troisième personne, qui exprime de la part du sujet un certain recul par rapport aux événements présentés et donc un certain "détachement " à l'égard de la réalité.

Le récit schizophrénique dans lequel l'héroïne se divise d'avec ellemême, dans lequel le clivage du moi est manifesté, agit en quelque

22 Jean-Claude Pariente, op. cit., p. 59.

23 Vincent Descombes, l'Inconscient malgré lui, Paris, Minuit, 1977, p. 139. 
sorte comme un champ de bataille ${ }^{24}$. Ce dédoublement du moi en une partie qui observe et une partie qui est observée ${ }^{25}$ est le résultat d'un conflit entre le "je" et cet autre, par l'entremise duquel est effectuée la prise en charge des expériences vécues. C'est par l'instance de la troisième personne que l'héroïne parvient à se délivrer du joug de son passé, de ces morceaux de temps mort qui lui collent au dos comme une boue glacée (p. 16). Si l'autre est le lieu où le refoulé fait retour, où le sujet se dédouble, où la réalité est mise en cause, c'est dans cette instance que le sujet trouve sa place signifiante ${ }^{26}$, où il se constitue. Compte tenu de ce qui précède, nous dirions donc que ce n'est pas du sujet d'énonciation que découle l'intérêt du discours schizophrénique, mais plutôt de cet écart entre le sujet qui parle (sujet d'énonciation) et le sujet dont il parle (sujet de l'énoncé).

\section{Pour un modèle d'énonciation du récit schizophrénique}

Dire que c'est par l'entremise de glissements de la première à la troisième personne que l'héroöne réussit à se distancier de son moi de jadis ne nous apprendra rien d'important, à moins de déterminer la valeur de cet écart et de décrire les mécanismes par lesquels l'héroïne effectue son jeu de subversion. Si l'on va au-delà du message que le récit schizophrénique nous communique, c'est-à-dire le désir de se distancier de certaines expériences traumatisantes, le refus de faire face aux motifs de son aliénation psychique et la tendance à nier la réalité en cause, on peut montrer que le récit schizophrénique déploie un modèle d'énonciation spécifique. C'est en recourant aux principales composantes de l'acte d'énonciation (le locuteur et les données spatio-temporelles) que nous tenterons d'élaborer un modèle d'énonciation du récit schizophrénique. Même si nous acceptons que c'est avant tout dans le jeu des glissements pronominaux que sévit, chez l'héroïne, ce conflit entre le "je" et cet autre qui menace à tout moment de l'absorber et de l'aspirer, nous croyons néanmoins que le flottement des instances narratives entraîne d'autres ambiguïtés qui doivent être prises en charge pour que l'instance schizophrénique soit saisie dans sa totalité.

Qui dit schizophrénie dit dépersonnalisation momentanée 27 , dédoublement de la personnalité, clivage du moi. Qui dit schizophrénie dit également désinvestissement de la réalité, combat avec le réel et même mise à mort $d u$ réel ${ }^{28}$. En parlant de soi à la troisième personne, Gabrielle abolit un point de repère qui est essentiel à son combat avec le réel, son instance narcissique: le point focal des repères spatio-temporels. Tout en traduisant de la part de l'héroïne un désir

24 Paul-Claude Racamier, op. cit., p. 80.

25 J. Pontalis et J.-B. Laplanche, op. cit., p. 68.

26 Jacques Lacan, Écrits, Paris, Seuil, 1966, p. 431 et 689.

27 Paul-Claude Racamier, op. cit., p. 72.

28 Ibid., p. 104 et 49. 
de se distancier de certaines expériences vécues, tout en signalant une certaine perte $d u$ sens $d u$ réel ${ }^{29}$, les glissements de la première à la troisième personne servent à projeter dans le récit de Gabrielle un non-je (débrayage actantiel) et à postuler un non-ici (débrayage spatial) et un non-maintenant (débrayage temporel) ${ }^{30}$. Bref, en se dédoublant, l'héroïne effectue non seulement un certain détachement personnel, mais également spatio-temporel à l'endroit de la réalité ${ }^{31}$.

Par la présence de nombreux segments rétrospectifs faisant l'objet de multiples occurrences ou d'évocations partielles inachevées, le récit de Gabrielle a l'apparence d'un vaste mouvement de va-et-vient dans lequel les souvenirs se succèdent en désordre. En plus de se présenter comme une série de petits tableaux fragmentaires sans suite apparente, le récit schizophrénique met en scène d'autres ambiguités qui ne peuvent être négligées. En étant presque entièrement dénué de toutes références spatio-temporelles, de tout embrayeur susceptible de préciser le temps et le lieu d'énonciation du discours schizophrénique, le récit de l'héroïne se trouve privé de l'ancrage référentiel fondamental ${ }^{32}$ à toute activité énonciative.

Au cours de son récit, l'héroïne ne précise jamais où elle se situe lors de l'évocation de ses souvenirs: l'instance spatiale du discours schizophrénique n'est jamais explicitement désignée. À l'exception de quelques remarques: Pourtant je ne suis pas vieille. Non. J'ai trente ans (p. 16), Gabrielle a neuf ans et elle a la peau duveteuse (p. 17), le récit ne met en scène aucun indicateur susceptible de préciser le temps de l'énonciation. Nous retrouvons également dans le récit_de Gabrielle de nombreux adverbes qui n'apportent aucune indication temporelle précise dans la mesure où l'instance de discours où ils sont produits ${ }^{33}$ n'est jamais spécifiée: c'était plus tard c'était le lendemain de rien ou. c'était hier le temps s'était dissous dans cette forme de néant [...] j'étais convaincue à ce moment que j'étais cadavre. (p. 191. Nous soulignons) Tout au long d'Après la boue, le moment et le lieu à partir desquels l'héroïne énonce son récit schizophrénique ne sont jamais précisés.

Il ressort de ce qui précède qu'au discours schizophrénique correspond un "énoncé type " dans lequel les indicateurs de la deixis visent plutôt à expulser hors de l'instance d'énonciation [...] les catégories de la personne, de l'espace et du temps ${ }^{34}$, qu'à effectuer un retour à l'instance d'énonciation. Les déictiques qui servent normalement à préciser

29 Paul-Claude Racamier, op. cit., p. 118.

30 A. Greimas et J. Courtés, Sémiotique. Dictionnaire raisonné de la théorie du langage, Paris, Hachette, 1979, p. 79.

31 J. Pontalis et J.-B. Laplanche, op. cit., p. 433.

32 André Joly, "Sur l'acte d'énonciation. À propos d'un fragment de discours interieur", Bulletin de la société de stylistique anglaise, $n^{\circ} 3,1981, p .19$.

33 Émile Benveniste, op. cit., p. 262.

34 A. Greimas et J. Courtés, op. cit., p. 119. 
qui parle, le lieu où ça parle et le temps dont ça parle ${ }^{35}$, fonctionnent différemment dans le discours de Gabrielle. Comme nous avons essayé de l'indiquer par cette structure tripartite, le récit schizophrénique semble être réglé par un processus de débrayage qui vise à le dépouiller des éléments fondamentaux à la reconstitution de l'instance d'énonciation:

\begin{tabular}{|c|c|c|}
\hline débrayage actantiel & - & $\begin{array}{l}\text { la non-personne (elle/Gabrielle) } \\
\text { regarder toujours, se voir spectacle } \\
\text { de soi dans sa tête (p. 16) }\end{array}$ \\
\hline \multirow[t]{2}{*}{ débrayage spatial } & - & le $n o n-i c i$ \\
\hline & & $\begin{array}{l}\text { brutalement projetée au centre } \\
\text { d'elle-même [...] dans les eaux } \\
\text { noires de son passé (p. } 75)\end{array}$ \\
\hline \multirow[t]{2}{*}{ débrayage temporel } & - & le non-maintenant \\
\hline & & $\begin{array}{l}\text { le temps s'était dissous dans cette } \\
\text { forme de néant (p. 191) }\end{array}$ \\
\hline
\end{tabular}

Dans tous les fragments de récit dans lesquels l'héroïne se voi[t] spectacle de soi dans sa tête (p. 16), les changements de formes pronominales ainsi que l'imprécision du contexte spatio-temporel créent, sur le plan de la narration, des ambiguïtés qui ne sont jamais entièrement résolues. Par la pluralité des instances narratives, l'anonymat de l'interlocuteur, les incohérences diégétiques et la disposition atemporelle des fragments rétrospectifs, le récit schizophrénique opère une division de l'unité diégétique ${ }^{36}$ qui met en cause la centralité du "je". La présentation de l'héroïne comme la prisonnière d'un rêve perpétuel (p. 175) dans lequel la réalité et l'illusion se chevauchent et se confondent, met en cause l'autorité du sujet énonciateur ainsi que la fonction du récit comme un simple instrument d'effets de réel. Le jeu des glissements déictiques auquel se prête la narratrice sert peut-être à marquer chez l'héroïne l'acheminement vers la folie; toutefois, ce qui importe, c'est de reconnaître que par l'entremise de ce discours schizophrénique, le roman de La Rocque semble mettre en question de nombreux éléments fondamentaux de toute cuvre romanesque: le statut du récit, la notion de personnage, le cadre de l'action, la fonction du narrataire, la relation entre le narrateur et le personnage. À l'instar de nombreuses œuvres modernes, c'est dans une certaine mesure en vue de piéger la vraisemblance, de gommer la représentation et de dissoudre le sujet ${ }^{37}$ que ce jeu de glissements déictiques fut inscrit au sein d'Après la boue.

35 Jean-Michel Adam, Linguistique et discours littéraire, Paris, Larousse, 1976, p. 316.

36 Jean Ricardou, le Nouveau Roman, Paris, Seuil, 1978, p. 139.

37 Sylvère Lotringer, "Une révolution romanesque», Nouveau roman: hier, aujourd'hui, tome I, Paris, U.G.E., 1972, p. 328. 